\title{
L'instabilite Rotulienne Objective (A Propos de Quatre Cas)
}

\author{
M. J. El Mekkaoui", J. Boukhris, T. El Yacoubi, M. Bousaidane, B. Chafry, D. Bencheba, A. S. Bouabid, M. Boussouga
}

Service de Traumatologie-Orthopédie II - H.M.I. Mohammed V - Rabat, Morocco

DOI: $\underline{10.36347 / \text { sasjs.2020.v06i04.011 }}$

| Received: 30.03 .2020 | Accepted: 06.04.2020 | Published: 27.04 .2020

*Corresponding author: Mohamed Jalal El Mekkaoui

Abstract

Original Research Article

Objective patellar instability is a pathology which, without being rare is not very common. It concerns the teenager and the young adult, with a female prevalence. This pathology is the consequence of multiple factors of an osseous nature, capsulo-ligamentaires and muscular. It gathers two clinical entities: repeating luxation and traumatic luxation followed by disorders. By this study, we report the analysis of the medical files of four patients who are taken in charge by the service of Traumatology-Orthopedy II of the HMIMV of Reduction, for an objective patellar instability during 2 years (2018-2019). We made an approach of this affection, so as to show through the achieved results the importance of a precise radiological analysis in the choice of a surgical strategy, associating osseous gestures and others on the soft parts while holding account of the age of the patients and the degree of the dysfunction. The forecast is dominated by the appearance of a femoro-patellar osteoarthritis at an early age.

Keywords: Instability - Patella - Radiography - Surgery.

Copyright @ 2020: This is an open-access article distributed under the terms of the Creative Commons Attribution license which permits unrestricted use, distribution, and reproduction in any medium for non-commercial use (NonCommercial, or CC-BY-NC) provided the original author and source are credited.

\section{INTRODUCTION}

L'instabilité rotulienne objective est une pathologie qui, sans être rare est peu commune, c'est l'apanage de l'adolescent et l'adulte jeune, avec une prédominance féminine, elle est la conséquence de facteurs multiples d'ordre osseux, capsuloligamentaires et musculaires. Elle regroupe deux entités cliniques: la luxation récidivante et la luxation traumatique suivi de troubles.

Notre étude vise à attirer l'attention sur l'importance d'une analyse radiologique précise dans le choix d'une stratégie chirurgicale, associant des gestes osseux et d'autres sur les parties molles, tout en tenant compte de l'âge des patients et du degré du dysfonctionnement.

\section{MATERIELS ET METHODES}

Nous rapportons une étude rétrospective portant sur une série de 4 cas d'instabilité rotulienne objective, colligés au service entre 2018 et 2019.

Le recul moyen était de 1 ans. Nos patients se répartissaient en 2 femmes et 2 hommes, dont l'âge moyen était de 22 ans avec des extrêmes de 16 ans et 28 ans. Un antécédent d'une luxation rotulienne a été retrouvé chez tous les patients (plusieurs épisodes chez certains). L'examen clinique des genoux a retrouvé une amyotrophie du quadriceps chez les 4 patients, choc rotulien chez 3, signes du rabot et de Smilie positifs chez les 4 . Le bilan radiologique a objectivé une bascule et subluxation rotulienne chez les 4 patients, un signe de croisement chez 3 patients, une ostéochondrite du condyle externe et rotule basse chez un patient (Fig1). La TDM a montré une TAGT élevée (supérieure à $20 \mathrm{~mm}$ en extension et $14 \mathrm{~mm}$ en flexion) chez 2 patients, la bascule rotulienne manifeste chez tous les patients et la dysplasie de la trochlée chez 2 patients (Fig-2). Le geste chirurgical s'est résumé à une section de l'aileron externe (Ficat externe) et une plastie d'Insall chez 2 patients; chez les 2 autres, ils ont en plus bénéficié d'une médialisation avec abaissement et avancement de la TTA (Fig 3 \& 4). Les résultats étaient très satisfaisants avec une rotule stable, indolore et reprise libre des activités.

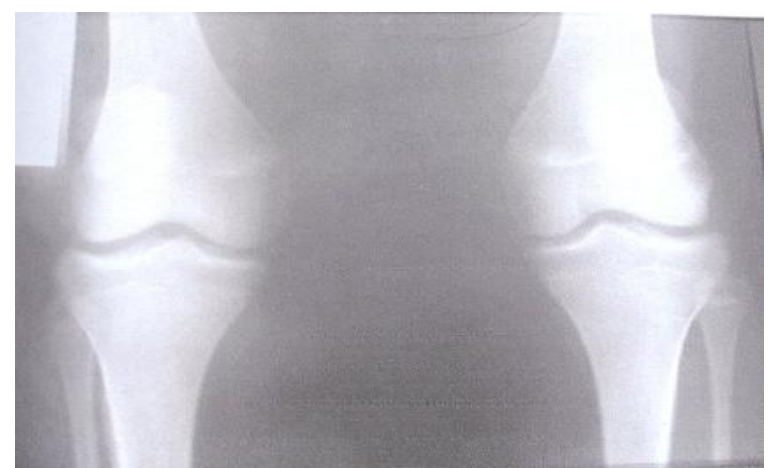

Fig-1: Radiographie de profil des genoux montrant une divergeance rotulienne 


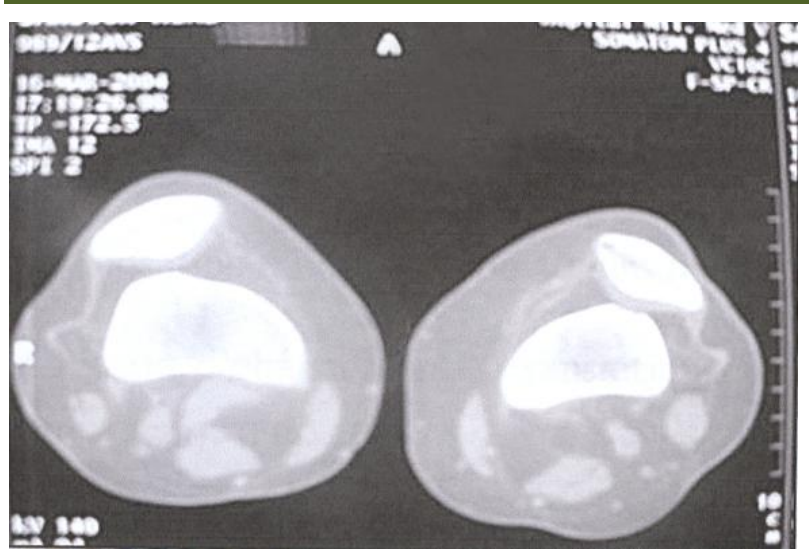

Fig-2: TDM du genou gauche montrant une bascule et subluxation de la rotule

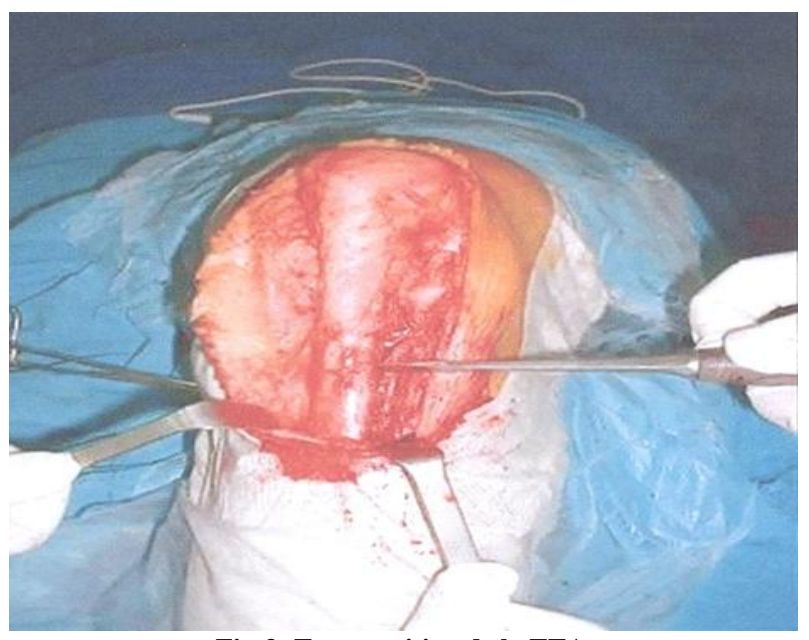

Fig-3: Transposition de la TTA

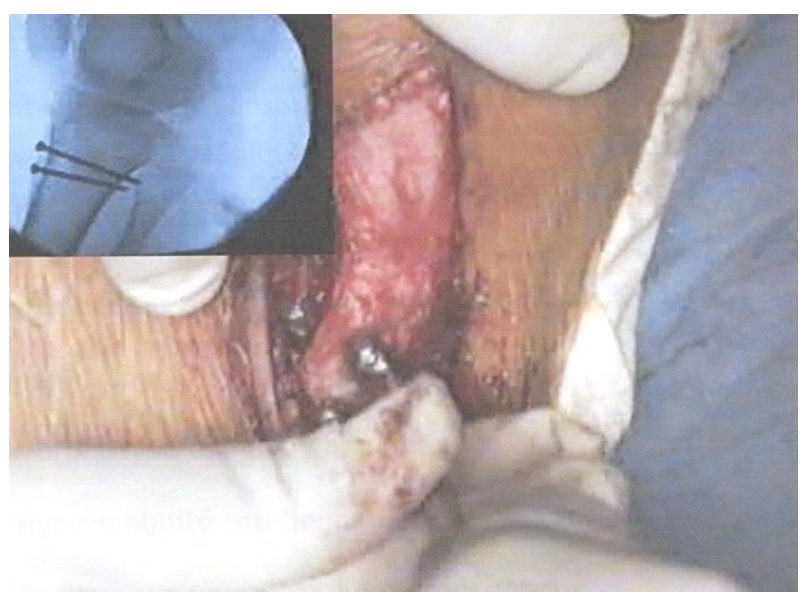

Fig-4: Mobilisation interne de la TTA fixée par 2 vis

\section{DISCUSSION}

L'incidence de l'instabilité rotulienne objective, n'est pas bien connue, dans notre formation, nous avons noté une admission annuelle de 2 cas, alors que les autres séries rapportent une moyenne de 3,5 cas [1-3]. L'âge moyen de nos patients est de 22 ans (16-28 ans), ce qui concorde avec la littérature qui rapporte un âge moyen de 23 ans, avec des extrêmes d'âge de 14 à 50 ans [4-6]. La composante génétique de cette pathologie est bien connue [7], une forme familiale de luxation récidivante de la rotule a été rapportée dans la littérature.

L'instabilité rotulienne objective, se distingue sur le plan clinique par la clarté des signes fonctionnels: un épisode de luxation rotulienne diagnostiqué ou traité, suivi quelques mois plus tard d'un syndrome rotulien manifeste et de plus en plus gênant [4]. Plusieurs épisodes de luxations rotuliennes extrêmes vraies de plus en plus faciles à réduire par le malade lui-même [4].

La radiologie standard permet pour l'œil averti, un diagnostic de certitude dans la majorité des cas quand il s'agit d'une luxation suivie de trouble. Les travaux de Dejour [10], Caton et Dechamps [8, 9], Goutalier et Bernageau [10] et bien d'autres sont d'un excellent appoint et permettent un bilan très satisfaisant de la maladie, néanmoins, la TDM reste irremplaçable pour la mesure de la TA-GT et de l'angle de la bascule rotulienne. A l'instar d'autres études, nous pensons que le scanner est impératif en préopératoire, pour le diagnostic positif, la radiographie standard suffit [4-8].

Le traitement des instabilités rotuliennes objectives est chirurgical. Il s'adresse à tous les malades demandeurs. La section de l'aileron externe (intervention de Ficat) est un geste constamment pratiqué, cependant, il est reconnu de façon unanime que ce geste pratiqué isolément n'est pas apte à traiter une instabilité rotulienne, les taux de récidive observés pouvant aller jusqu'à $40 \%$ (11). Ce geste a été le temps T1 chez tous nos patients quand il existe une TA-GT pathologique (> $14 \mathrm{~mm}$ en extension et/ou $12 \mathrm{~mm}$ en flexion), la TTTA devient impérative.

Nous avons opté pour la technique de la baguette pédiculée fixée et sur la possibilité de débuter précocement la rééducation.

Il s'emble se concrétiser un consessus sur le fait que les plasties osseuses doivent être vivement réfléchies, et proposées dans les cas graves, et leurs éventuelles complications doivent être bien expliquées aux malades.

Avec un recul moyen de 12 mois, tous les patients sont satisfaits, sur le plan subjectif avec, disparition des douleurs et de l'appréhension, disparition des luxations interactives. Sur le plan objectif, il n'y avait pas eu de rotule basse postopératoire iatrogène. La consolidation de la TTA était obtenue chez tous les patients. Les résultats de notre série peuvent être considérés, compte tenu du niveau d'activité de nos malades (un seul sportif avéré), comme excellents. Ce fait apparaît largement dans la littérature ou l'instabilité rotulienne objective reste la pathologie rotulienne d'ordre chirurgical par excellence [4-11]. 


\section{CONCLUSION}

L'instabilité rotulienne objective est une pathologie peu commune, sans corrélation anatomoclinique, ainsi le bilan radiologique reste l'élément clé dans le diagnostic, permettant ainsi de rechercher des anomalies anatomiques caractéristiques. La TDM permet par ailleurs de préciser différents facteurs nécessaires pour adopter l'indication chirurgical et pour contrôler les résultats, sachant que l'instabilité rotulienne objective relève d'un possible acte chirurgical seul capable de stabiliser la rotule. Le pronostic est dominé peu l'apparition d'une arthrose fémoro-patellaire à un âge précoce.

\section{REFERENCES}

1. Goutallier D, Bernageau J, Lecudonnec B. The measurement of the tibial tuberosity. Patella groove distanced technique and results (author's transl). Revue de chirurgie orthopedique et reparatrice de l'appareil moteur. 1978;64(5):423-8.

2. Judet J, Jude H. L'allongement du vaste externe dans les luxations et subluxations de la rotule. Nouv Press Méd, 1975;4:22.

3. Goutallier D, Blachier D, Norotte G, Bernageau J. Etude des différents facteurs influençant les résultats du traitement des instabilités rotuliennes avec luxations (s). Rhumatologie (Aix-les-Bains). 1993;45(5):101-3.

4. Dejour H. Instabilité de la rotule. Encycl Med Chir. 1996;14-328-1-10.

5. Bensahel H, Souchet PH, Pennecot GF, DESGRIPPES Y, FOURRIER M. Instabilités rotuliennes de l'enfant. Discussion. Chirurgie (Paris). 1994;120(5):298-300.

6. Conte J. Rôle de la tomodensitométrie dans l'étude des instabilités rotuliennes: Genou-RachisMédecine du sport et football. Kinésithérapie scientifique. 2001(411):17-9.

7. Dejour H, Dejour D. Souffrance et instabilité rotulienne. Cahiers d'enseignement de la SOFCOT. 1999;71:67-70.

8. Dejour H, Dejour D. Les instabilités rotuliennes. Cahiers d'enseignement de la SOFCOT. 1999;71:71-82.

9. Garin C. L'instabilité rotulienne chez l'enfant. Cahiers d'enseignement de la SOFCOT. 1995;52:203-17.

10. Goutallier D, Bernageau J. Le point sur la TA-GT. Cahiers d'enseignement de la SOFCOT. 1999;71:175-82.

11. Chassaing V, Tremoulet J. Luxation de la rotule: techniques chirurgicales et indications. Journal de traumatologie du sport. 2000;17(2):74-82. 